\title{
A dioxaborine cyanine dye as a photoluminescence probe for sensing carbon nanotubes
}

\author{
Mohammed Al Araimi ${ }^{*} 1,2$, Petro Lutsyk ${ }^{1,3}$, Anatoly Verbitsky ${ }^{3}$, Yuri Piryatinski ${ }^{3}$, \\ Mykola Shandura ${ }^{4}$ and Aleksey Rozhin ${ }^{* 2}$
}

\section{Full Research Paper}

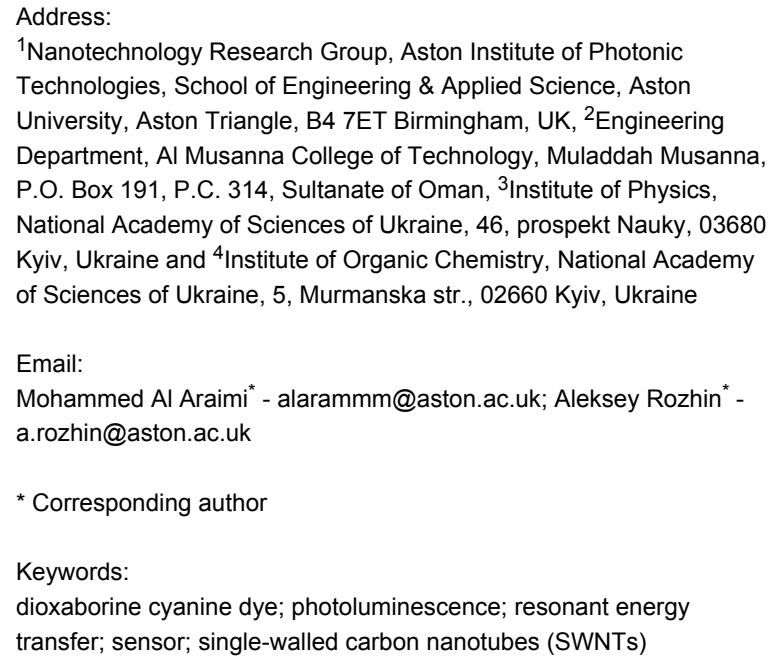

Beilstein J. Nanotechnol. 2016, 7, 1991-1999. doi:10.3762/bjnano.7.190

Received: 30 July 2016

Accepted: 30 November 2016

Published: 14 December 2016

This article is part of the Thematic Series "Functional materials for environmental sensors and energy systems".

Guest Editor: M. Penza

(C) 2016 Al Araimi et al.; licensee Beilstein-Institut. License and terms: see end of document.

\begin{abstract}
The unique properties of carbon nanotubes have made them the material of choice for many current and future industrial applications. As a consequence of the increasing development of nanotechnology, carbon nanotubes show potential threat to health and environment. Therefore, development of efficient method for detection of carbon nanotubes is required. In this work, we have studied the interaction of indopentamethinedioxaborine dye (DOB-719) and single-walled carbon nanotubes (SWNTs) using absorption and photoluminescence (PL) spectroscopy. In the mixture of the dye and the SWNTs we have revealed new optical features in the spectral range of the intrinsic excitation of the dye due to resonance energy transfer from DOB-719 to SWNTs. Specifically, we have observed an emergence of new PL peaks at the excitation wavelength of $735 \mathrm{~nm}$ and a redshift of the intrinsic PL peaks of SWNT emission (up to $40 \mathrm{~nm}$ ) in the near-infrared range. The possible mechanism of the interaction between DOB-719 and SWNTs has been proposed. Thus, it can be concluded that DOB-719 dye has promising applications for designing efficient and tailorable optical probes for the detection of SWNTs.
\end{abstract}

\section{Introduction}

Carbon nanotubes exhibit unique physical and chemical properties distinctive from other materials because of their extreme aspect ratio offering a number of exciting applications in rein- forced plastics, conductive composites, sensors and photonic devices [1]. However, with the rapid growth of nanotechnology, carbon nanotubes pose a significant potential threat to health 
and environment in the near future due to their toxicity [1-5]. Therefore, efficient sensing techniques for nanotubes have to be developed before the harmful exposure of carbon nanotubes to ambient environment.

One of the most efficient and versatile techniques of chemical sensing is photoluminescence (PL) detection [6] involving a substantial increase of the PL emission due to the presence of target molecules, i.e., carbon nanotubes. The amplification of the PL signal from single-walled carbon nanotubes (SWNTs) can be obtained via covalent formation of $\mathrm{sp}^{3}$-defects on the tube surface [7], encapsulation of small organic molecules inside the SWNTs [8] and non-covalent interaction of the tube sidewall with $\pi$-conjugated organic compounds [9-12]. Considering sensors, particular attention has to be paid to the PL enhancement in aqueous media, like the complexation and resonant energy transfer (RET) from cyanine dyes to the SWNTs covered by anionic surfactants in water [12]. However, a drawback of these cyanine-based systems is the RET in the range of PL excitation wavelengths, where the SWNTs have substantial intrinsic emission. As a result of the RET, PL intensity is amplified relatively to the intrinsic emission of SWNTs, so the complexation effect has limited relative sensitivity.

In this paper, we focused on studies of an interaction of SWNTs with the indopentamethinedioxaborine dye (DOB-719) having an extended $\pi$-conjugated system (Figure 1 ) and absorption in near infrared (NIR) range. The choice of the dye is driven by its unique interaction with the SWNTs resulting in the emergence of new PL peaks in the range of excitation wavelength at 650-780 nm, where the PL emission of the SWNTs is very low. Generally, polymethine dyes with a terminal dioxaborine group have intense PL signals, efficient two-photon absorption, high hyperpolarizability [13], and features of effective PL probes for amines and ammonia [14-16]. Optical properties (absorption and PL) of monomeric and dimeric forms of DOB-719 were reported in [16] showing that there is a weak interaction of SWNTs with DOB-719. The novelty of the present study is an elucidation of the interaction of the dye with the SWNTs providing clear evidences of RET from DOB-719 to SWNTs. The

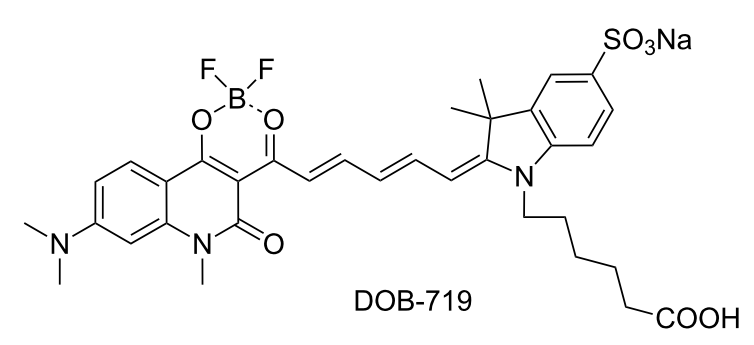

Figure 1: The chemical structure of DOB-719. new results obtained in these studies allow us to propose the mechanism of interaction between the SWNT and DOB-719, where the dye is attracted to the SWNT surface via $\pi-\pi$ stacking with the hydrophobic part while the hydrophilic groups are facing the aqueous medium.

\section{Results and Discussion}

We have studied the interaction of DOB-719 with SWNTs by spectral analysis of absorption and PL for as-prepared and aged (measured $24 \mathrm{~h}$ after the mixing) mixtures of DOB-719 with SWNTs in water. Essentially, an admixture of the dye $(0.001 \mathrm{mg} / \mathrm{mL})$ to SWNTs dispersed with sodium dodecylbenzenesulfonate (SDBS) resulted in the emergence of several new spectroscopic features compared to neat dye and neat SWNTs. Figure 2 shows the PL excitation-emission (PLE) maps, with the $X$-axis representing the emission wavelengths $\left(\lambda_{\mathrm{EM}}\right)$ and the $Y$-axis representing the excitation wavelengths $\left(\lambda_{\mathrm{EX}}\right)$ for neat SWNTs (Figure 2a) and as-prepared mixtures of DOB-719 with SWNTs (Figure $2 \mathrm{~b}$ ). In Figure $2 \mathrm{a}$, the predominant $(6,5),(7,5)$, and $(8,4)$ chiralities of the SWNTs are clearly seen in the range of $\lambda_{\mathrm{EX}}$ at $\mathrm{E}_{22}, \mathrm{E}_{33}$, and $\mathrm{E}_{44}(300-800 \mathrm{~nm})$ and $\lambda_{\mathrm{EM}}$ at $\mathrm{E}_{11}$ $(950-1350 \mathrm{~nm})[17,18]$. In Figure 2b, the same PL features of the predominant SWNT chiralities are supplemented with new peaks in the range of $\lambda_{\mathrm{EX}}=650-780 \mathrm{~nm}$ (with maxima at $\lambda_{\mathrm{EX}}=$ $735 \mathrm{~nm}$ ) and $\lambda_{\text {EM }}$ corresponding to the $\mathrm{E}_{11}$ SWNT emission levels. The new features in the PLE maps for the mixtures of DOB-719 with the SWNTs appear because of the formation of a nanostructured complex of the dye with the SWNT. A similar complexation phenomenon has been demonstrated recently for systems consisting of SWNTs and the cyanine dye astraphloxin $[12,19]$. However, the present system is different (not only because of the emergence of new PL peaks in the range of $\lambda_{\mathrm{EX}}=650-780 \mathrm{~nm}$ ) because of a strong redshift of the intrinsic SWNT peaks in the $\lambda_{\mathrm{EM}}$ (Table 1) and the quenching of these SWNT emission peaks. The strong redshifts for the $\lambda_{\mathrm{EM}}$ maxima of the mixtures in comparison with the neat nanotubes for all chiralities are summarised in Table 1. The variations of redshifts for different SWNT chiralities can be explained by a structural matching of the $\pi$-electron systems of SWNTs with particular chirality and the developed $\pi$-electron system of DOB-719. The different redshifts provide not only sensitivity, but also selectivity of the PL detection towards the nanotube diameters.

The energy diagram shown at the right-hand side of Figure 2a represents a two-component system consisting of the anionic surfactant SDBS and the SWNTs in water, where the anionic surfactant forms micelles around the nanotubes having typical exciton energy levels of PL excitation at $\mathrm{E}_{22}$ and emission at $E_{11}$. In Figure $2 b$, the three-component system is modelled, where DOB-719 is attached to the SWNT surface via $\pi-\pi$ 
a)

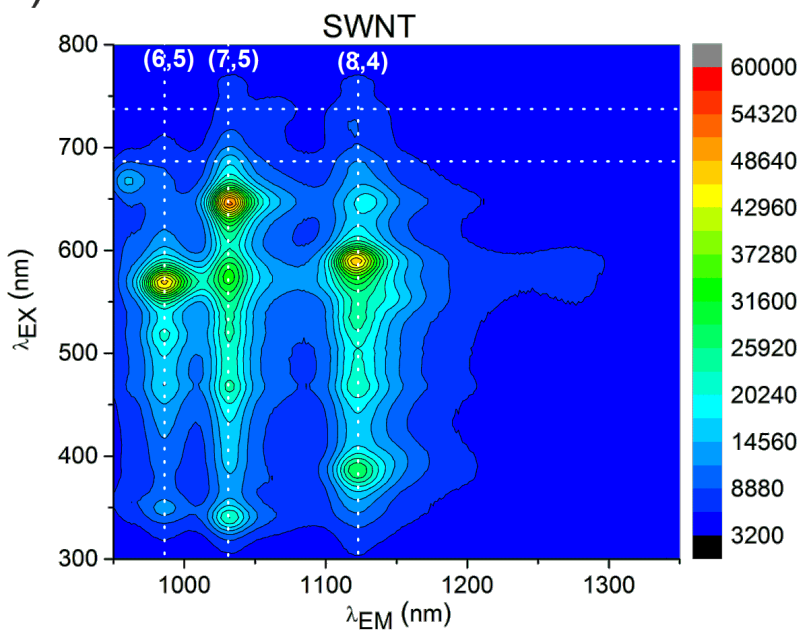

b)

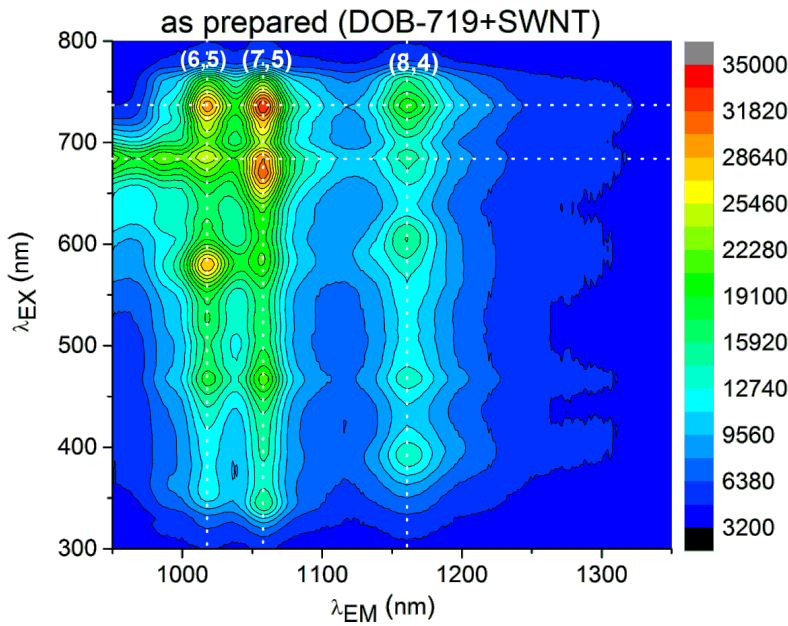

\section{SWNT (SDBS)}

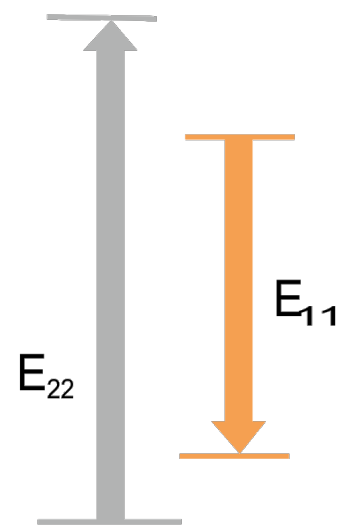

DOB-719+SWNT (SDBS)

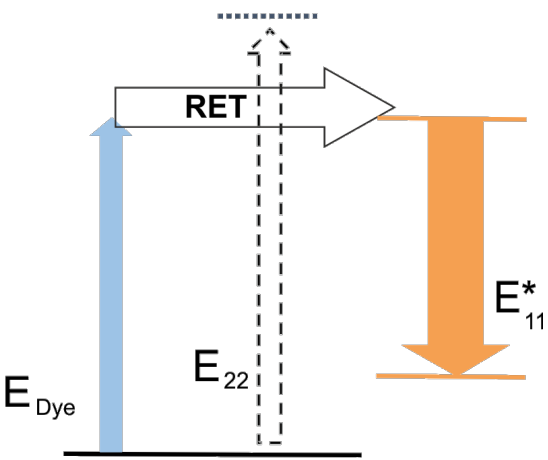

Figure 2: PLE maps (left) and the energy diagrams (right) for (a) dispersion of neat SWNTs and (b) as-prepared mixture of DOB-719 with the SWNT in water. Vertical dashed lines indicate $\lambda_{E M}$ positions of $E_{11}$ for SWNT of $(6,5),(7,5)$, and $(8,4)$ chirality, horizontal dashed lines indicate the position of new PL peaks at $\lambda_{E X}=735 \mathrm{~nm}$ (the RET from DOB-719 to the SWNT) and the position of DOB-719 monomers maximum at $\lambda_{E X}=685 \mathrm{~nm}$. High PL intensities are coded in red colour, whereas low intensities are coded in blue colour. The concentration of DOB-719 in panel (b) is $0.001 \mathrm{mg} / \mathrm{mL}$.

\begin{tabular}{|c|c|c|c|}
\hline \multirow[b]{2}{*}{ SWNT chirality } & \multicolumn{3}{|c|}{$\lambda_{\mathrm{EM}}\left(\mathrm{E}_{11}\right.$ or $\left.\mathrm{E}_{11}{ }^{*}\right)$} \\
\hline & dispersion of the SWNT & $\begin{array}{l}\text { as-prepared mixture of } \\
\text { DOB-719 and the SWNT }\end{array}$ & redshift \\
\hline $\begin{array}{l}(6,5) \\
\text { at } \lambda_{\mathrm{EX}}=570 \mathrm{~nm}\end{array}$ & $985 \mathrm{~nm}(1.258 \mathrm{eV})$ & $1018 \mathrm{~nm}(1.217 \mathrm{eV})$ & $33 \mathrm{~nm}(41 \mathrm{meV})$ \\
\hline $\begin{array}{l}(7,5) \\
\text { at } \lambda_{\mathrm{EX}}=650 \mathrm{~nm}\end{array}$ & $1032 \mathrm{~nm}(1.201 \mathrm{eV})$ & 1057 nm (1.172 eV) & $25 \mathrm{~nm}(29 \mathrm{meV})$ \\
\hline $\begin{array}{l}(8,4) \\
\text { at } \lambda_{\mathrm{EX}}=590 \mathrm{~nm}\end{array}$ & $1121 \mathrm{~nm}(1.105 \mathrm{eV})$ & $1161 \mathrm{~nm}(1.067 \mathrm{eV})$ & $40 \mathrm{~nm}(38 \mathrm{meV})$ \\
\hline
\end{tabular}


stacking of the hydrophobic parts ( $\pi$-conjugated frame) while the aqueous medium is faced by the hydrophilic parts $\left(\mathrm{SO}_{3}{ }^{-}\right.$ and $\mathrm{COO}^{-}$). The non-covalent attachment of the dye to the nanotube results in the efficient RET at the excitation wavelength in the range of 700-760 $\mathrm{nm}$ and a strong redshift of the $\mathrm{E}_{11}$ levels (to $\mathrm{E}^{*}{ }_{11}$ ) in the NIR range (Table 1). The excitation energy of DOB-719 in this range $\left(E_{\text {Dye }}\right)$ is transferred to the SWNT levels $\left(E^{*}{ }_{11}\right)$ via the RET. This way, DOB-719 (donor of energy) and SWNT (acceptor of energy) form nanostructured complexes via non-covalent interaction. As a result, the diagrams model the emergence of new PL peaks due to formation of non-covalent complexes of DOB-719 with the SWNT. The complexes of such dyes have high potential to be used as an effective PL probe for the detection of carbon nanotubes considering that there is clear dependence of the RET response on the SWNT concentration (see Supporting Information File 1, Figure S1).

Figure 3 shows the absorption spectra for the mixture of DOB719 with the SWNT in comparison with its components. To understand the nature of the new PL features (in the range of $\lambda_{\mathrm{EX}}=650-780 \mathrm{~nm}$ ) we have estimated the absorption peak positions using Gaussian deconvolution. The absorption of the neat SWNT in the range of 300-800 nm (Figure 3a, curve 1) exhibits low intensity $E_{22}$ and $E_{33}$ excitonic transitions [20]. The neat DOB-719 (Figure 3a, curve 2) has a maximum absorption at $687 \mathrm{~nm}(1.80 \mathrm{eV})$ featuring monomers (free molecules) of the dye in aqueous solution [16]. The second peak has vibrational $(631 \mathrm{~nm} ; 1.96 \mathrm{eV})$ and dimeric $(623 \mathrm{~nm} ; 1.99 \mathrm{eV})$ components for DOB-719 molecules as shown and discussed in Figure S2 (Supporting Information File 1). The absorption spectrum of as-prepared mixture of DOB-719 with SWNTs (Figure 3a, curve 3) has two maxima at $692 \mathrm{~nm}(1.79 \mathrm{eV})$ and $742 \mathrm{~nm}(1.67 \mathrm{eV})$, where the former peak is a signature of the dye monomers. The latter peak correspond to the dye molecules associated with the SWNTs and is redshifted by $50 \mathrm{~nm}$ comparing to the absorption maximum of monomeric peak [12]. The intensity ratio of these peaks allows us to monitor both the amount of the dye monomers in the mixture and the amount of the dye associated with the SWNTs. Roquelet et al. reported a similar behavior for SWNT-porphyrin complexes, where an overlapping $20 \mathrm{~nm}$ split in the porphyrin (Soret) band was observed [21]. Here, the emergence of the $50 \mathrm{~nm}$ redshifted peak in the mixtures hinders a strong overlap with the monomer absorption band. The absorption spectrum of the aged mixture (measured $24 \mathrm{~h}$ after mixing, Figure 3a, curve 4) has only one maximum at $742 \mathrm{~nm}(1.67 \mathrm{eV})$ and a short wavelength shoulder (peak after Gaussian deconvolution at $700 \mathrm{~nm}(1.77 \mathrm{eV})$ ). Thus, the relative contribution of absorption for the dye-SWNT complexes in the aged mixtures is much higher comparing to the as-prepared mixture.

The two-component mixture of SDBS and DOB-719 was studied resulting in no significant change of the absorption (Supporting Information File 1, Figure S3, curve 3) and PL spectra of the dye in the presence of SDBS. There is no evidence for the interaction between the surfactant (at premicellar and micellar concentrations) and the dye. Referring to our previous studies on the interaction of SDBS and astraphloxin (a polymethine dye with cyanines at both terminal groups) resulting in the aggregation of the dye. There is no such behaviour in the case of DOB-719.

In addition, we have studied the three-component system of DOB-719, SDBS, and SWNTs, where an extra amount of surfactant was added (see Supporting Information File 1, Figures S3-S5) to obtain a concentration of SDBS above the critical micellar concentration of $0.15 \mathrm{mg} / \mathrm{mL}$ [22]. The three-compo- a)

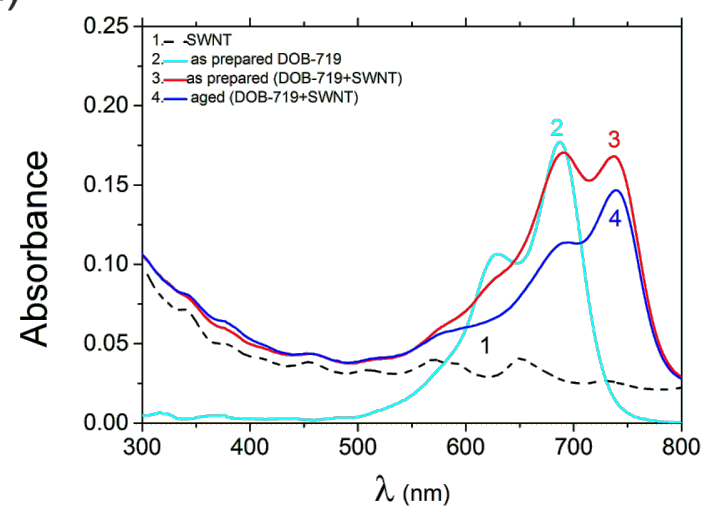

b)

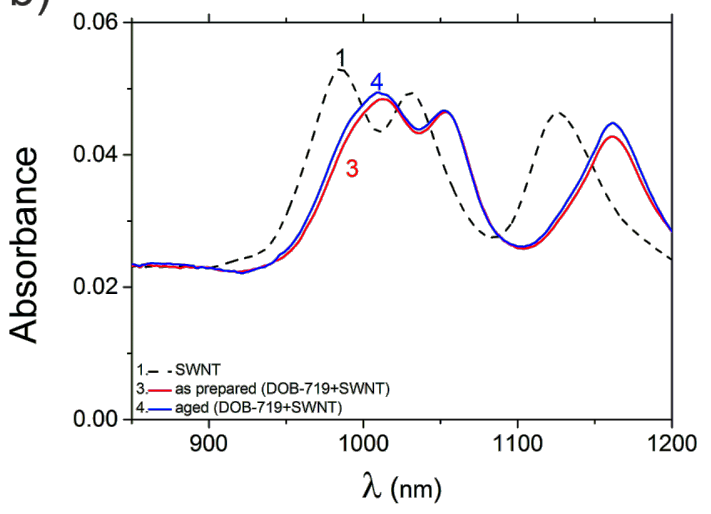

Figure 3: Absorption spectra in (a) the visible and (b) the NIR range for dispersions of SWNT (1), aqueous solutions of neat DOB-719 (2), as-prepared mixtures of DOB-719 with SWNT (3), and aged mixtures of DOB-719 with SWNT (4). The concentration of DOB-719 is $0.001 \mathrm{mg} / \mathrm{mL}$. 
nent mixture at micellar concentration of SDBS does not exhibit new bands in the range of 700-780 nm (in absorption and PL excitation). Thus, no RET from DOB-719 is possible when the micelles of SDBS fully cover the SWNTs. This shows another dissimilarity of the studied system in comparison with SWNT-SDBS-astraphloxin, where the RET was equally efficient at micellar concentrations of SDBS [19].

The absorption of the neat SWNTs in the range of 850-1200 nm exhibits $E_{11}$ excitonic peaks [20] at $\lambda=986$, 1031, and $1127 \mathrm{~nm}$ (Table 2; Figure 3b, curve 1). As a result of the dye admixture to the SWNTs, the $\mathrm{E}_{11}$ peaks are red-shifted in comparison to neat SWNTs (Table 2, Figure 3b). However, the $E_{11}$ peaks of the mixtures practically do not change due to the aging (Figure $3 \mathrm{~b}$, curves 3 and 4 ). The $\mathrm{E}_{i i}$ optical transition energies of the SWNTs strongly depend on the dielectric constant of both the nanotubes and the background of their surroundings $\left(\varepsilon_{\mathrm{bg}}\right)$. Dielectric-screening effects become particularly evident when the SWNTs dispersed in liquids are compared to the nanotubes in air [23], so an increase of $\varepsilon_{\text {bg }}$ results in a redshift of the $E_{i i}$ transition energies [23-25]. On one hand, in our work, the maximum and the minimum shifts in $\mathrm{E}_{11}$ exciton energy are 32 and $25 \mathrm{meV}$, respectively (Table 2), which are lower than the average shifts of $\mathrm{E}_{11}=55 \mathrm{meV}$ obtained in [23]. On the other hand, the redshifts in Table 2 are much lower than the redshift (of a few millielectronvolts) achieved when the surfactant around the nanotubes is diluted [25]. In the complex of DOB-719 with SWNT, the local increase of $\varepsilon_{\mathrm{bg}}$ can result from (i) a better access of water to the SWNTs and/or (ii) polar groups of the dye getting in close proximity to the nanotube surface. Thus, the nanotube transitions at $\mathrm{E}_{11}$ show significant redshifts, which are attributed to increase of $\varepsilon_{\text {bg }}$ around the nanotubes due to the presence of the dye molecules.

To provide better insight on the new spectral features in Figure 2 and Figure 3, we extracted the PL emission and excitation spectra for the as-prepared and aged mixtures from the PLE maps (Figure 2 and Figure S6, Supporting Information File 1). The PL emission spectra (Figure 4a) were extracted at $\lambda_{\mathrm{EX}}=$ $735 \mathrm{~nm}$ attributed to the complexation of DOB-719 with the SWNTs. The excitation spectra (Figure $4 \mathrm{~b}-\mathrm{d}$ ) for the as-pre- pared and aged mixtures were extracted at $\lambda_{\mathrm{EX}}=1016,1059$, and $1161 \mathrm{~nm}$ corresponding to the $(6,5),(7,5)$, and $(8,4)$ chiralities, respectively. For neat SWNTs the excitation spectra were extracted at $\lambda_{\mathrm{EX}}=985,1032$, and $1121 \mathrm{~nm}$ referring to the $(6,5),(7,5)$, and $(8,4)$ chiralities, respectively. Importantly, the PL intensities for the $(6,5)$ and $(7,5)$ chiralities at $\lambda_{\mathrm{EX}}=735 \mathrm{~nm}$ have increased (approximately five-fold) for the as-prepared mixtures comparing those of neat SWNTs. However, the PL intensity for the $(8,4)$ chirality at $\lambda_{\mathrm{EX}}=735 \mathrm{~nm}$ has grown approximately 2.5 -times for the as prepared mixtures. This indicates that the RET from the dye attached to the $(6,5)$ and $(7,5)$ chiralities SWNT is more efficient than the RET for the $(8,4)$ chirality. Thus, we can well discriminate the excitation peaks from excitonic levels of SWNTs and the RET from the dye complexed to the SWNT, where the RET has maximum at $\lambda_{\mathrm{EX}}=735 \mathrm{~nm}$. Finally, the ageing of the mixtures affects severely the complexes formed with SWNTs of $(7,5)$ chirality (Figure $4 \mathrm{a}, \mathrm{c})$, whereas the complexes of $(6,5)$ and $(8,4)$ chirality are quite stable.

In Figure 2 and Figure 4, one can notice that, as a result of admixing DOB-719 to the SWNTs, the PL intensity of the intrinsic SWNT emission was quenched significantly for all chiralities (ca. $40 \%$ at $\lambda_{\mathrm{EX}}=570 \mathrm{~nm}, \lambda_{\mathrm{EM}}=985 \mathrm{~nm}$ for $(6,5)$; ca. $40 \%$ at $\lambda_{\mathrm{EX}}=650 \mathrm{~nm}, \lambda_{\mathrm{EM}}=1032 \mathrm{~nm}$ for $(7,5)$; ca. $70 \%$ at $\lambda_{\mathrm{EX}}=590 \mathrm{~nm}, \lambda_{\mathrm{EM}}=1121 \mathrm{~nm}$ for $\left.(8,4)\right)$. This finding indicates that the interaction of DOB-719 with the SWNTs alters the intrinsic PL properties of the nanotubes. However, in the complexation system of astraphloxin-SWNT, intrinsic PL peaks of the SWNTs remained unchanged [12]. The moderate quenching of intrinsic SWNT emission evidences direct interaction of DOB-719 and the SWNT, which in case of the astraphloxin-based systems is restricted by the presence of the surfactant. Assumingly, SDBS does not affect the interaction of DOB-719 and the SWNT.

To understand the nature of the changes in the mixtures, the PLE maps in the emission range of the dye were analysed (Figure 5). We have put together the PLE maps for the as-prepared and aged samples of both neat DOB-719 (Figure 5a,b) and the mixture of DOB-719 with the SWNT (Figure 5c,d) registered in the range of $\lambda_{\mathrm{EX}}=400-750 \mathrm{~nm}$ and $\lambda_{\mathrm{EM}}=$

Table 2: Peaks of absorption $\left(E_{11}\right)$ for the neat SWNT dispersions and the as prepared mixtures of DOB-719 with the SWNT, redshifts of $E_{11}$ in absorption due to an admixture of the dye.

$\begin{array}{llll}\begin{array}{l}\text { predominant chirality of } E_{11} \text { peaks } \\ {[17]}\end{array} & \text { dispersion of the SWNTs } & \begin{array}{l}\text { as-prepared mixture of DOB-719 } \\ \text { and the SWNT }\end{array} & \text { redshift } \\ (6,5) & 986 \mathrm{~nm}(1.257 \mathrm{eV}) & 1011 \mathrm{~nm}(1.225 \mathrm{eV}) & 25 \mathrm{~nm}(32 \mathrm{meV}) \\ (7,5) & 1031 \mathrm{~nm}(1.202 \mathrm{eV}) & 1053 \mathrm{~nm}(1.177 \mathrm{eV}) & 22 \mathrm{~nm}(25 \mathrm{meV}) \\ (8,4) & 1127 \mathrm{~nm}(1.100 \mathrm{eV}) & 1161 \mathrm{~nm}(1.068 \mathrm{eV}) & 34 \mathrm{~nm}(32 \mathrm{meV})\end{array}$


a)

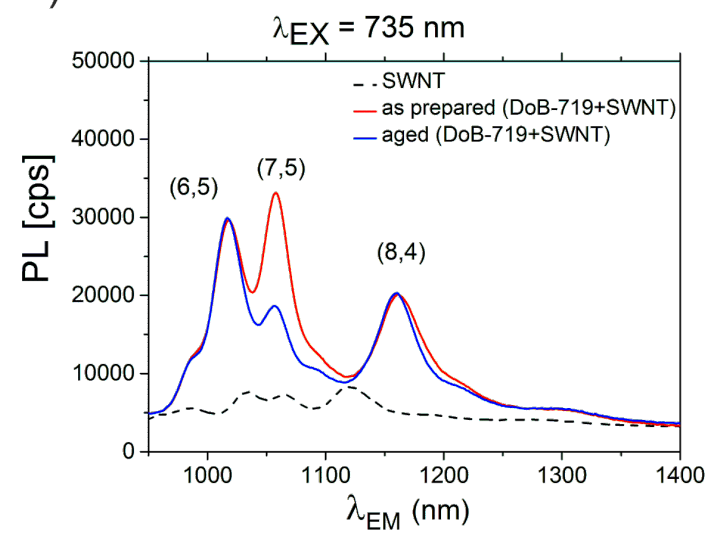

c)

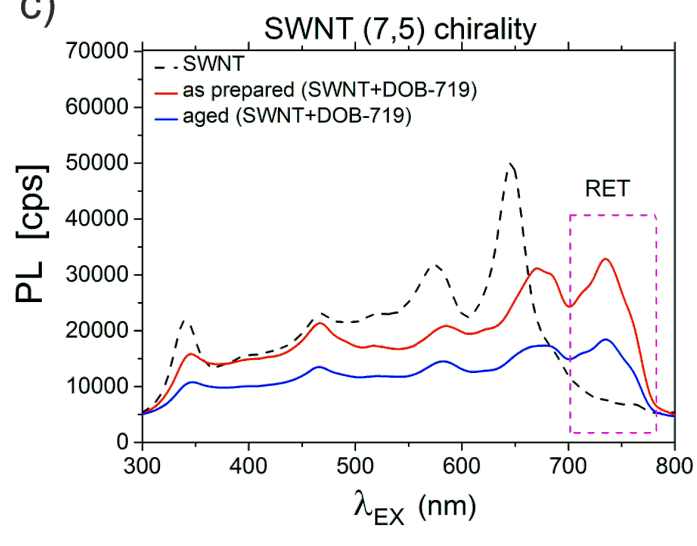

b)

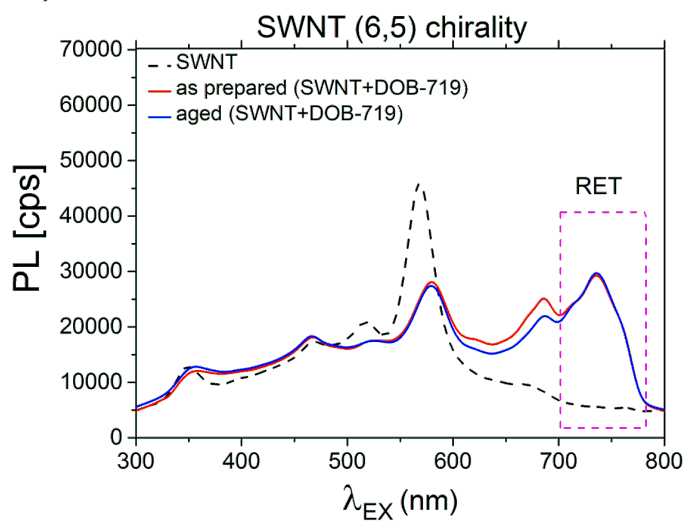

d)

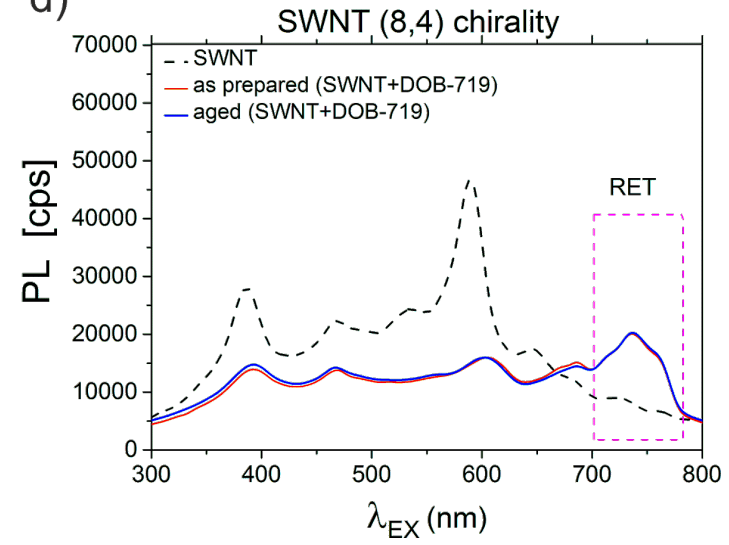

Figure 4: PL spectra for the as-prepared (red curves) and aged (blue curves) mixtures of DOB-719 and SWNTs and dispersions of neat SWNTs (dashed curves) in water. (a) PL emission spectra at $\lambda_{\mathrm{EX}}=735 \mathrm{~nm}$ and PL excitation spectra (b) $(6,5),(\mathrm{c})(7,5)$, and (d) $(8,4)$ chiralities. PL spectra for the as-prepared and aged mixtures are extracted at $\lambda_{\mathrm{EM}}=1016,1059$, and $1161 \mathrm{~nm}$ for the $(6,5),(7,5)$, and $(8,4)$ chiralities, respectively. PL spectra for neat SWNTs are extracted at $\lambda_{E M}=985,1032$, and $1121 \mathrm{~nm}$ for the $(6,5),(7,5)$, and $(8,4)$, respectively. The RET from the dye to the SWNT at $\lambda_{\mathrm{EX}}=735 \mathrm{~nm}$ is indicated in panels $\mathrm{b}-\mathrm{d}$.

600-800 nm. The PL peak with $\lambda_{\mathrm{EX}}=685 \mathrm{~nm}$ and $\lambda_{\mathrm{EM}}=$ $720 \mathrm{~nm}$ (Figure 5) correlates with the monomeric peak in the absorption spectra at $687 \mathrm{~nm}$ (Figure 3a and Supporting Information File 1, Figure S2). The second band with weak PL in the range of $\lambda_{\mathrm{EX}}=615-640 \mathrm{~nm}$ and $\lambda_{\mathrm{EM}}=700-725 \mathrm{~nm}$ (in Figure $5 \mathrm{a}, \mathrm{c}, \mathrm{d}$ ) could be related to both the dimeric PL of DOB$719\left(\lambda_{\mathrm{abs}}=623 \mathrm{~nm}\right)$ and $/$ or a second vibrational transition of the monomers $\left(\lambda_{\mathrm{abs}}=631 \mathrm{~nm}\right)$ discussed in Supporting Information File 1 in Figure S2. In Figure 5b, the PL intensity of the monomeric peak (with $\lambda_{\mathrm{EX}}=685 \mathrm{~nm}$ and $\lambda_{\mathrm{EM}}=720 \mathrm{~nm}$ ) for aged neat DOB-719 is quenched (to approx. a half) and a new band with strong PL appears. This band has a maximum at $\lambda_{\mathrm{EX}}=575 \mathrm{~nm}$ and $\lambda_{\mathrm{EM}}=680 \mathrm{~nm}$, and its intensity is about four times higher than that of the as-prepared sample. In this spectral range, the as-prepared dye has only a weak and featureless shoulder (Figure 5a). The above new band and the low-intensity band in the range of $\lambda_{\mathrm{EX}}=400-450 \mathrm{~nm}$ and $\lambda_{\mathrm{EM}}=$ 660-700 nm (Figure 5b) correspond to the new bands of absorption developed with time at $\lambda=335-600 \mathrm{~nm}$ (Supporting
Information File 1, Figure S7, curves 2 and 3). These new features in the aged samples can be associated with the product of the dioxaborine cycle hydrolysis [13].

The PLE maps in the dye emission range for the mixtures show that the PL intensity of the monomeric peak (at $\lambda_{\mathrm{EX}}=685 \mathrm{~nm}$ and $\lambda_{\mathrm{EM}}=720 \mathrm{~nm}$ ) is quenched down to about a third for both the as-prepared (Figure 5a,c) and the aged (Figure 5b,d) mixtures. The quenching of the monomeric peak due to the ageing is almost the same (down to about a half) for the neat dye (Figure 5a,b) and for the mixtures with the SWNT (Figure $5 \mathrm{c}, \mathrm{d}$ ). Importantly, the PL intensity of the band at $\lambda_{\mathrm{EX}}=$ $575 \mathrm{~nm}$ and $\lambda_{\mathrm{EM}}=680 \mathrm{~nm}$ (resulted from the hydrolysis of dioxaborine cycle) does not change significantly after ageing of the mixture (Figure 5c,d). As it has been discussed before, in the neat dye the same band grew about four-fold during the ageing process (Figure 5a,b). This result indicates that the molecules of DOB-719 associated in the complex with the SWNTs are more stable towards hydrolysis, whereas the free dye mole- 

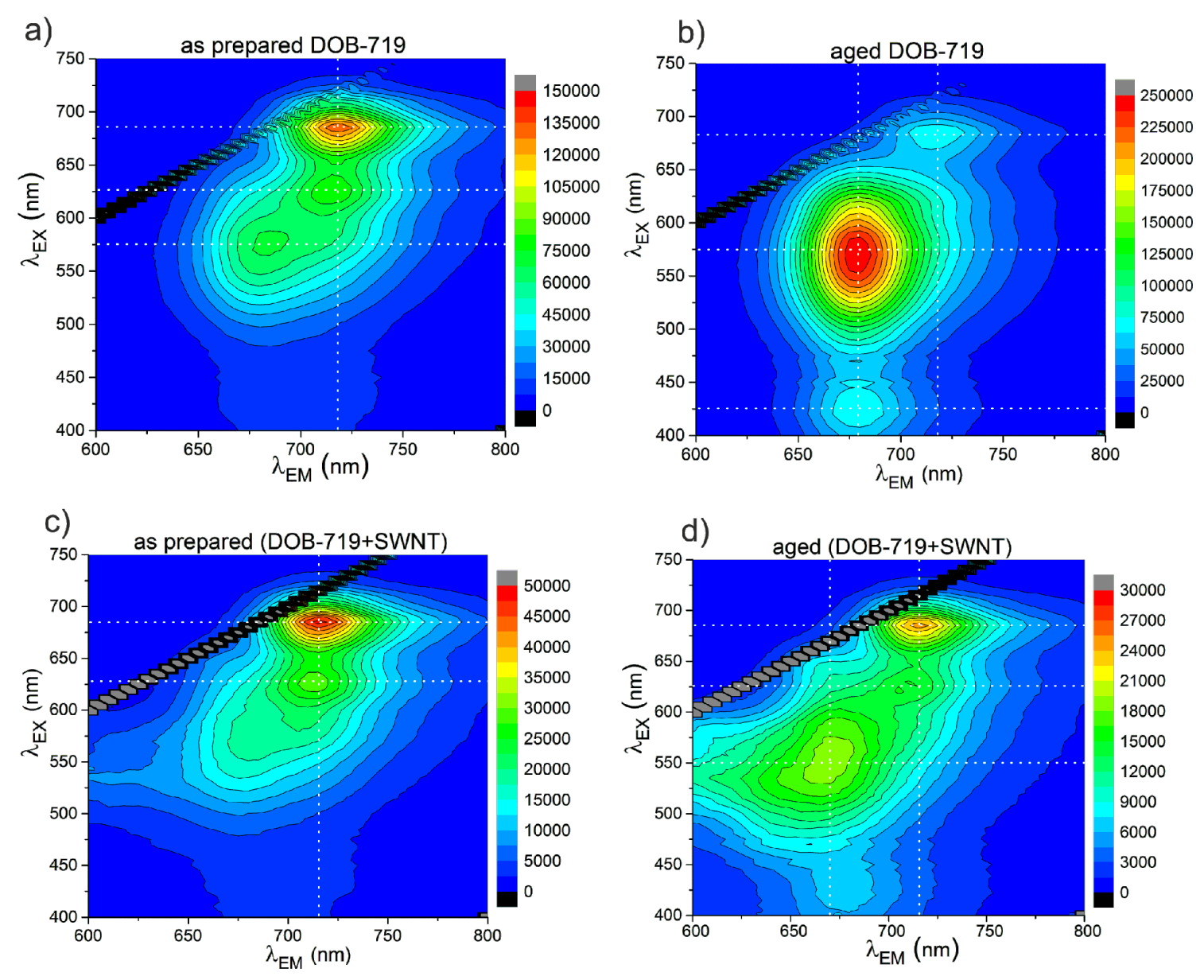

Figure 5: PLE maps of aqueous solutions for (a) the as-prepared and (b) aged neat DOB-719 as well as (c) the as-prepared and (d) aged mixtures of DOB-719 with SWNTs in water. High PL intensities are coded in red colour, whereas low intensities are coded in blue colour. The concentration of DOB-719 is $0.001 \mathrm{mg} / \mathrm{mL}$.

cules (monomers) degrade over time. This effect is supported by the spectral change in the absorption of the as-prepared and the aged mixtures of DOB-719 with SWNTs (Figure 3a, curves 3 and 4) showing a higher stability for the peak at $\lambda_{\mathrm{abs}}=742 \mathrm{~nm}$ of the complexes than the monomeric peak at $\lambda_{\mathrm{abs}}=692 \mathrm{~nm}$ (Figure 3a, curves 4).

Thus, the mechanism of PL quenching for DOB-719 and the SWNTs is similar to the astraphloxin system [12], as the quenching of both dyes in the mixtures is approximately the same. Particularly, comparing our systems of SWNTs-surfactant-dye with the system of SWNTs-porphyrin without surfactant, which demonstrated much stronger quenching (ca. 1000 times) [21]. Therefore, in the SWNTs-SDBS-DOB-719 complexes the surfactant molecules could remain between the dye and the nanotubes. However, the direct contact of the DOB-719 with the SWNTs is also possible. In fact, the distance between DOB-719 and the SWNTs has to be much smaller (or overlap of their $\pi$-conjugated systems is stronger) than between the
SWNTs and astraphloxin [12]. This statement is supported by a much stronger redshift of the $\mathrm{E}_{11}$ peak (22-40 meV vs $6 \mathrm{meV}$ [12]), evident quenching of the intrinsic PL emission of the SWNTs, and no interaction of the dye with SDBS in the complexes of SWNT-SDBS-DOB-719 in comparison with the system of SWNT-SDBS-astraphloxin [12,19].

\section{Conclusion}

We have studied the interaction of the organic dye DOB-719 with SWNTs dispersed by an anionic surfactant (SDBS) in water using absorption and PL spectroscopy. We have found that DOB-719 is associated with the SWNTs and forms nanostructured complexes via $\pi-\pi$ stacking by the hydrophobic conjugated frame facing the aqueous medium with the hydrophilic groups $\mathrm{SO}_{3}{ }^{-}$and $\mathrm{COO}^{-}$. The interaction is evidenced by new optical features in the range of the intrinsic excitation of the dye because of resonant energy transfer from the dye to the SWNTs. In the mixtures of DOB-719 with SWNTs, a new absorption peak corresponding to the dye molecules associated 
to the SWNTs has emerged being $50 \mathrm{~nm}$ redshifted in comparison to the monomeric peak of neat DOB-719 absorption. The new PL peaks featuring the RET have appeared at the excitation wavelength $735 \mathrm{~nm}$ and the emission wavelength corresponding to SWNT $E_{11}$ emission levels. Moreover, the $\lambda_{\mathrm{EM}}$ of PL peaks for the mixtures are strongly redshifted (up to $40 \mathrm{~nm}$ ) in comparison with the peaks of the neat nanotubes for all the SWNT chiralities evidencing strong dielectric screening of the formed complexes. The new peak developing at the excitation wavelength $735 \mathrm{~nm}$ can be used for PL detection of SWNTs in aqueous environment. In the mixture of DOB-719 and SWNTs, the monomeric dye molecules were either in free form and degraded severely, or associated in a complex with the SWNT micelles and became more stable. Thus, the interaction between DOB-719 and the SWNT opens a new way to design efficient and tailorable optical probes for not only sensitivity, but also selectivity of the PL detection towards the nanotube diameters.

\section{Experimental}

\section{Sample preparation \\ Synthesis DOB-719}

DOB-719 was synthesised as shown in Scheme 1 by the reaction of ( $2 \mathrm{mmol}$ ) dioxaborine 1 [13] with (2.2 mmol) of hemicyanine 2 [26] and $(2.2 \mathrm{mmol})$ of triethylamine (TEA). The resultant mixture was stirred at room temperature in $6 \mathrm{~mL}$ of pyridine for $3 \mathrm{~h}$. After vacuum distillation of the reaction mixture, the residue was grinded with isopropyl alcohol (iPrOH) and filtered out. The product was dissolved in $80 \%$ aqueous ethanol and then the solution of sodium acetate $(0.2 \mathrm{~g})$ in ethanol was added. The precipitate was filtered out and purified via chromatography on a silica column $(7: 3 \mathrm{v} / \mathrm{v}$ acetone/methanol as an eluent) to yield $300 \mathrm{mg}(20 \%)$ of product as a green powder. ${ }^{1} \mathrm{H}$ NMR (300 MHz, DMSO- $\left.d_{6}\right) \delta 1.3-1.36(\mathrm{~m}, 2 \mathrm{H}$, $\left.\mathrm{CH}_{2}\right), 1.54-1.63\left(\mathrm{~m}, 10 \mathrm{H}, 2 \mathrm{CH}_{3}+2 \mathrm{CH}_{2}\right), 2.09-2.13(\mathrm{~m}, 2 \mathrm{H}$, $\left.\mathrm{CH}_{2}\right), 3.14\left(\mathrm{~s}, 6 \mathrm{H}, \mathrm{N}\left(\mathrm{CH}_{3}\right)_{2}\right), 3.54\left(\mathrm{~s}, 3 \mathrm{H}, \mathrm{NCH}_{3}\right), 3.94-3.98$ $\left(\mathrm{m}, 2 \mathrm{H}, \mathrm{CH}_{2}\right), 6.19(\mathrm{~d}, J=15 \mathrm{~Hz}, 1 \mathrm{H}), 6.34(\mathrm{~s}, 1 \mathrm{H}), 6.52$ (t, $J=$ $15 \mathrm{~Hz}, 1 \mathrm{H}), 6.78$ (d, $J=9 \mathrm{~Hz}, 1 \mathrm{H}), 7.19$ (d, $J=9 \mathrm{~Hz}, 1 \mathrm{H}), 7.58$ (d, $J=6 \mathrm{~Hz}), 7.68-7.73(\mathrm{~m}, 2 \mathrm{H}), 7.87(\mathrm{~d}, J=9 \mathrm{~Hz}, 1 \mathrm{H}), 8.13(\mathrm{t}$, $J=12 \mathrm{~Hz}, 1 \mathrm{H}), 8.29$ (t, $J=12 \mathrm{~Hz}, 1 \mathrm{H})$.

\section{Preparation of SWNTs}

SWNTs were prepared in a similar manner as described in [12], where purified SWNTs (CoMoCAT) were used to prepare the SWNT dispersions. $1.2 \mathrm{mg}$ of the SWNTs were dispersed in $20 \mathrm{~mL}$ of deionized (DI) water in the presence of $6.5 \mathrm{mg}$ sodium dodecylbenzene sulfonate (SDBS). We selected SDBS among other ionic surfactants because of its high efficiency in dispersing SWNTs [27,28]. The above dispersions were exposed to ultrasonication (NanoRuptor, Diagenode) for $1 \mathrm{~h}$ at $21 \mathrm{kHz}$ and $250 \mathrm{~W}$. Then, the dispersions were subjected to ultracentrifugation for $3 \mathrm{~h}$ at $17^{\circ} \mathrm{C}$ at $45000 \mathrm{rpm}$ (Beckman Coulter Optima Max-XP, MLS 50 rotor) to remove the aggregate phase and obtain the supernatant solutions of debundled SWNTs. The top $70 \%$ of the dispersion is then decanted. To prepare mixture of DOB-719, we used $20 \%$ of the initial SWNT dispersion with SDBS to maintain the dye admixture with the SWNTs at the premicellar concentration $(0.065 \mathrm{mg} / \mathrm{mL})$. The origin and level of purity for all materials used in this manuscript are described in Supporting Information File 1.

\section{Experimental setup}

The absorption spectra in the visible and NIR ranges were measured using a Lambda 1050 UV-vis-NIR (Perkin Elmer) spectrometer with $1.5 \mathrm{~nm}$ increment. The deionized water was used as a reference in all measurements of the absorption spectra. The PL emission spectra at various excitation wavelengths were recorded using a Horiba NanoLog excitation-emission spectrofluorometer equipped with a InGaAs array detector cooled by liquid nitrogen and Si detector to produce PL excitation-emission maps (PLE maps), with the $X$-axis representing the emission wavelength $\left(\lambda_{\mathrm{EM}}\right)$ and the $Y$-axis representing the excitation wavelength ( $\left.\lambda_{\mathrm{EX}}\right)$. The PL measurements in the NIR were performed using entrance/exit slits of $14 \mathrm{~nm}$ in width for both the excitation and emission monochromators. Entrance/ exit of $2 \mathrm{~nm}$ slits were used for both the excitation and emission monochromators in the visible range measurements. All samples in this manuscript were measured under standard laboratory conditions. Also, the $\mathrm{pH}$ value was maintained at 7 to ensure a good dispersion of SWNTs and avoid bundling [29].

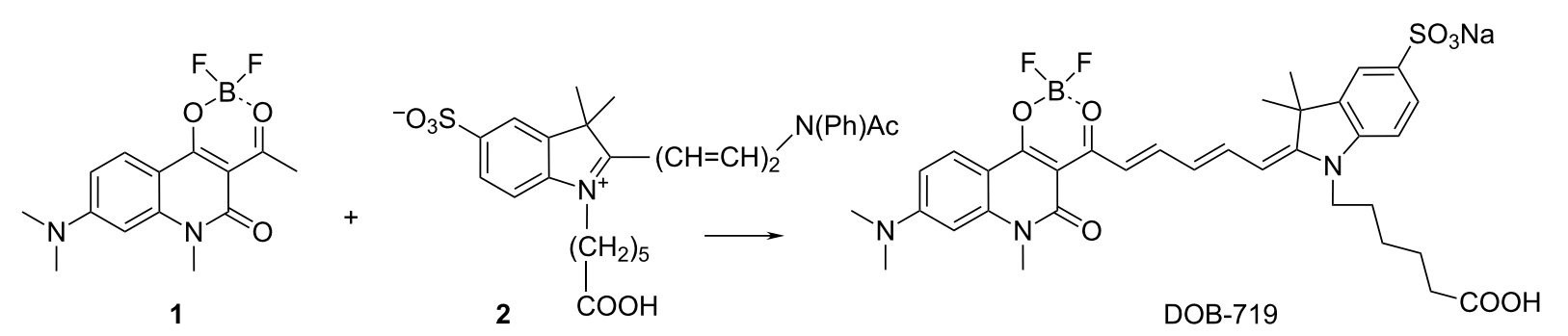




\section{Supporting Information}

\section{Supporting Information File 1}

Additional figures and discussion.

[http://www.beilstein-journals.org/bjnano/content/

supplementary/2190-4286-7-190-S1.pdf]

\section{Acknowledgements}

This work was supported by NATO SPS project (NUKR.SFPP \#984189) and EU FP 'Horizon- 2020' Marie Skłodowska-Curie Individual Fellowship (FOC4SIP, \#654733). M.A. acknowledges the support from the Ministry of Higher Education, Sultanate of Oman

\section{References}

1. De Volder, M. F. L.; Tawfick, S. H.; Baughman, R. H.; Hart, A. J. Science 2013, 339, 535-539. doi:10.1126/science.1222453

2. Lee, Y.; Geckeler, K. E. Adv. Mater. 2010, 22, 4076-4083. doi:10.1002/adma.201000746

3. Vardharajula, S.; Ali, S. Z.; Tiwari, P. M.; Eroğlu, E.; Vig, K.; Dennis, V. A.; Singh, S. R. Int. J. Nanomed. 2012, 7, 5361-5374. doi:10.2147/IJN.S35832

4. Hussain, S. M.; Braydich-Stolle, L. K.; Schrand, A. M.; Murdock, R. C.; Yu, K. O.; Mattie, D. M.; Schlager, J. J.; Terrones, M. Adv. Mater. 2009, 21, 1549-1559. doi:10.1002/adma.200801395

5. Kostarelos, K.; Bianco, A.; Prato, M. Nat. Nanotechnol. 2009, 4, 627-633. doi:10.1038/nnano.2009.241

6. Demchenko, A. P. Introduction to Fluorescence Sensing; Springer Science \& Business Media, 2008.

7. Piao, Y.; Meany, B.; Powell, L. R.; Valley, N.; Kwon, H.; Schatz, G. C.; Wang, Y. Nat. Chem. 2013, 5, 840-845. doi:10.1038/nchem.1711

8. Yanagi, K.; lakoubovskii, K.; Kazaoui, S.; Minami, N.; Maniwa, Y.; Miyata, Y.; Kataura, H. Phys. Rev. B 2006, 74, 155420. doi:10.1103/PhysRevB.74.155420

9. Ahmad, A.; Kern, K.; Balasubramanian, K. ChemPhysChem 2009, 10, 905-909. doi:10.1002/cphc.200800796

10. Ernst, F.; Heek, T.; Setaro, A.; Haag, R.; Reich, S. Adv. Funct. Mater. 2012, 22, 3921-3926. doi:10.1002/adfm.201200784

11. Vialla, F.; Delport, G.; Chassagneux, Y.; Roussignol, P.; Lauret, J. S.; Voisin, C. Nanoscale 2016, 8, 2326-2332. doi:10.1039/C5NR08023A

12. Lutsyk, P.; Arif, R.; Hruby, J.; Bukivskyi, A.; Vinijchuk, O.; Shandura, M.; Yakubovskyi, V.; Kovtun, Y.; Rance, G. A.; Fay, M.; Piryatinski, Y.; Kachkovsky, O.; Verbitsky, A.; Rozhin, A. Light: Sci. Appl. 2016, 5, e16028. doi:10.1038/lsa.2016.28

13. Gerasov, A. O.; Zyabrev, K. V.; Shandura, M. P.; Kovtun, Y. P. Dyes Pigm. 2011, 89, 76-85. doi:10.1016/j.dyepig.2010.09.007

14. Gerasov, A. O.; Shandura, M. P.; Kovtun, Y. P.; Vlasenko, Y. G.; Gorchev, V. F. J. Heterocycl. Chem. 2008, 45, 1665-1672. doi:10.1002/jhet.5570450617

15. Shandura, M. P.; Kovtun, Y. P.; Yakubovskyi, V. P.; Piryatinski, Y. P.; Lutsyk, P. M.; Perminov, R. J.; Verbitsky, A. B.; Rozhin, A. Key Eng. Mater. 2014, 605, 159-162. doi:10.4028/www.scientific.net/KEM.605.159
16. Shandura, M. P.; Kovtun, Yu. P.; Yakubovskyi, V. P.;

Piryatinski, Yu. P.; Lutsyk, P. M.; Perminov, R. J.; Arif, R. N.; Verbitsky, A. B.; Rozhin, A. Sens. Lett. 2014, 12, 1361-1367. doi:10.1166/sl.2014.3315

17. Bachilo, S. M.; Strano, M. S.; Kittrell, C.; Hauge, R. H.; Smalley, R. E.; Weisman, R. B. Science 2002, 298, 2361-2366.

doi:10.1126/science.1078727

18. Bachilo, S. M.; Balzano, L.; Herrera, J. E.; Pompeo, F.; Resasco, D. E.; Weisman, R. B. J. Am. Chem. Soc. 2003, 125, 11186-11187. doi:10.1021/ja036622c

19. Lutsyk, P.; Piryatinski, Y.; AlAraimi, M.; Arif, R.; Shandura, M.; Kachkovsky, O.; Verbitsky, A.; Rozhin, A. J. Phys. Chem. C 2016, 120, 20378-20386. doi:10.1021/acs.jpcc.6b06272

20. Weisman, R. B.; Bachilo, S. M. Nano Lett. 2003, 3, 1235-1238. doi:10.1021/nl034428i

21. Roquelet, C.; Langlois, B.; Vialla, F.; Garrot, D.; Lauret, J. S.; Voisin, C. Chem. Phys. 2013, 413, 45-54. doi:10.1016/j.chemphys.2012.09.004

22. Weiss, E.; Groenen-Serrano, K.; Savall, A. J. Appl. Electrochem. 2007, 37, 1337-1344. doi:10.1007/s10800-007-9367-4

23. Dyatlova, O. A.; Gomis-Bresco, J.; Malic, E.; Telg, H.; Maultzsch, J.; Zhong, G.; Geng, J.; Woggon, U. Phys. Rev. B 2012, 85, 245449. doi:10.1103/PhysRevB.85.245449

24. Ando, T. Physica E 2011, 43, 798-803. doi:10.1016/j.physe.2010.07.055

25. McDonald, T. J.; Engtrakul, C.; Jones, M.; Rumbles, G.; Heben, M. J. J. Phys. Chem. B 2006, 110, 25339-25346. doi:10.1021/jp065281x

26. Markova, L. I.; Fedyunyayeva, I. A.; Povrozin, Y. A.; Semenova, O. M.; Khabuseva, S. U.; Terpetschnig, E. A.; Patsenker, L. D. Dyes Pigm. 2013, 96, 535-546. doi:10.1016/j.dyepig.2012.09.007

27. Islam, M. F.; Rojas, E.; Bergey, D. M.; Johnson, A. T.; Yodh, A. G. Nano Lett. 2003, 3, 269-273. doi:10.1021/nl025924u

28. Tan, Y.; Resasco, D. E. J. Phys. Chem. B 2005, 109, 14454-14460. doi:10.1021/jp052217r

29. Duque, J. G.; Cognet, L.; Parra-Vasquez, A. N. G.; Nicholas, N.; Schmidt, H. K.; Pasquali, M. J. Am. Chem. Soc. 2008, 130, 2626-2633. doi:10.1021/ja0777234

\section{License and Terms}

This is an Open Access article under the terms of the Creative Commons Attribution License (http://creativecommons.org/licenses/by/4.0), which permits unrestricted use, distribution, and reproduction in any medium, provided the original work is properly cited.

The license is subject to the Beilstein Journal of Nanotechnology terms and conditions: (http://www.beilstein-journals.org/bjnano)

The definitive version of this article is the electronic one which can be found at: doi:10.3762/bjnano. 7.190 